\title{
The Meaningfulness of Consensus and Context in Diagnosing Evolvable Production Systems
}

\author{
Luis Ribeiro, José Barata, and João Ferreira \\ Universidade Nova de Lisboa, Faculdade de Ciências e Tecnologia, Campus da FCT-UNL, \\ Monte de Caparica 2829 - 516, Caparica, Portugal \\ $\{1 d r, j a b\} @ u n i n o v a . p t, j p f 19013 @ f c t . u n l . p t$
}

\begin{abstract}
An Evolvable Production System (EPS) is a complex and lively entity composed of intelligent modules that interact, through bio-inspired mechanisms, to ensure high system availability and seamless reconfiguration. The diagnosis of such dynamic systems, characterized by constant change, presents new diagnostic challenges and opportunities that can hardly be tackled by traditional approaches. On the one hand, given the decoupled nature of the system, fault interaction and propagation are harder to detect and contain, as is the development of a global diagnostic model, on the other hand local intelligence and careful characterization of the interactions, between the modules, can be explored to emerge the diagnostic functionalities. The impact of simple consensus mechanisms (majority voting) and fault context analysis (module and its current interactions states) is assessed in a multiagent-oriented application in the assembly domain to understand the validity and contribution of this approach in emerging useful self-diagnostic properties in EPS.
\end{abstract}

Keywords: Diagnosis, Evolvable Production Systems, Multi-agent Systems, Interaction Diagnosis, Hidden Markov Models.

\section{Introduction}

Mass Customization has been perceived as the excellence paradigm in industry and services however, as pointed out by [1], "we are now entering the era of network competition". This has led enterprises to seek innovative organizational paradigms [1, 2]: Supply chains, Extended Enterprises, Virtual Enterprises, Collaborative Networks, etc. In this context, industrial installations require improved agility to face the socioeconomic challenges ahead and effectively contribute to network competitiveness. Researchers in production paradigms, acknowledging this dynamic business environment, increasingly suggest and envision lively shop floors which are aggregations of intelligent and distributed interacting entities. Evolvable Production Systems (EPS) $[3,4]$ relying is such intelligent environments will allow quicker ramp-up times once both product design and shop-floor reconfiguration adjust to meet a balanced compromise These systems, however, are inherently complex and present significant diagnostic challenges and opportunities. Fault interaction and propagation are harder 
to detect and contain as it becomes more difficult to devise the diagnostic model of a system that, in the limit, can be in constant change and is highly heterogeneous. Nonetheless, local intelligence can be explored to circumvent these problems and enhance the diagnosis capabilities of the system by indirectly inferring the local state from the behaviour fault behaviour of other devices.

Being agile is also a matter of preventing breakdowns, incidents and developing sustainably. Intelligent automation such as required for future production systems must involve diagnostic capabilities that match the performance of the control solution.

Traditional diagnostic approaches often require a full model of the system and are not directly applicable to modern production paradigms like EPS. In fact, to avoid corrupt the control functionalities, the diagnostic system should be embedded in the paradigm and designed accordingly.

This has not been the case. Most diagnostic methods have been developed targeting a specific shop-floor component or a subsystem (manipulators, electrical motors, rotary equipment), or even the entire plant, rather than an evolving entity. The approach proposed in this paper is being developed under the framework of EPS whose control and design principles been investigated in the EUPASS ${ }^{1}$ project. Intelligent interaction is explored as a fundamental diagnostic feature in EPS. The main concept is the design of local (module level) diagnosers that evaluate their internal status is respect to a given interaction type taking into consideration their operational context and their sensorial perceptions. The objective is to, through this local interaction, emerge polarized consensus regarding disturbances in the shop floor. The presented experiments report some preliminary results of the ongoing research.

\section{Contribution to Technological Innovation}

Networked information systems are the cornerstone of today's society $[5,6]$. The pervasive nature of such system ensures seamless, and often unnoticed, everyday-life interaction. The natural evolution of these human-machine networks is towards complexity. This is manifested in the size of the network and in the interconnectivity degree. Industrial networks are not immune to this effect as the complexity of the installation grows more and more devices require communications and more processes need to be synchronized. There is a considerable set of industrial network standards that ease the integration of traditional logic based control devices. However, in the era of network competition, the flux of information between shop floor components and higher level management tools is increasingly important [7]. To meet these requirements there is an ongoing generalized research effort to change the nature and interfacing of the industrial devices in order to make them more pluggable and seamlessly available to other systems.

Research in this area will enable industry to effectively instantiate powerful production paradigms such as EPS at the cost of increased interaction complexity between the architectural building blocks (automation modules). As earlier detailed conventional shop floor diagnostic methods, alone, will not encompass this dramatic shift. This is the context addressed by the present work.

\footnotetext{
${ }^{1}$ EUPASS project http://www.eupass.org/
} 
The main contributions of the ongoing research can be summarized as follows: propose a diagnostic architecture that is suitable for intelligent automation system instantiating the EPS concept and in that context ensure the co-evolution of the diagnostic system while improving the overall sustainability of the installation; Capture the dynamics of fault propagation and interaction in distributed industrial systems composed of a high number of interaction modules and Enhance the traditional device-customized diagnostic approaches by introducing the network dimension and incorporating it in the local diagnosis.

\section{Related Literature}

A complete review on diagnostic methods derived from the automatic control community can be found in [8] where the application of: parameter estimation, evaluation of parity relations, state estimation and principal component analysis methodologies is properly covered.

A review of quantitative and qualitative history based methods where diagnosis is performed based on the previous system's faulty behaviour can be found in [9] where the application of artificial neural networks, probabilistic inference methods and expert system is discussed. Qualitative logic based diagnostic methods are covered in [10].

The research being reported in this paper, however, has a close connection with the authors' previous work developed under the Inlife Project ${ }^{2}$ where web services were applied to a pilot assembly cell and functionalities including self-monitoring/ diagnosis/reporting were implemented at device and process levels [11, 12]. A preliminary version of ACORDA [13], a prospective logic engine, that enables the revision of results, trough the encoding of preference rules was used. Although the engine provided an efficient platform to formalize the diagnostic models, the used version did not support modeling uncertainty. Probabilistic methods applied in the diagnosing industrial systems are reported in [14], [15] and in [16]. The first work proposes the application of Hidden Markov Models and principal component analysis to diagnose chemical processes. The second work uses Bayesian Networks to diagnose and study processes in a caravan manufacturing line. The third solution relies on a structured representation of the domain to tackle fault scenarios with multiple causes and fault propagation. The present work rather than considering the entire shop floor or subsystems focuses on the intelligent modules and their interactions with purpose of emerging at network level consistent fault propagation patterns.

\section{A Diagnostic Method Proposal}

The main goal of the proposed method is to, through stigmergy, emerge a coherent diagnostic perspective at network level. For that purpose each agent will evaluate its own internal state based on a sequence of observations that it performs as the fault event develops in the system. Each individual agent should be only able to observe its direct neighbours and its sensorial equipment. This constraint is to ensure that the

\footnotetext{
${ }^{2}$ InLife project http://www.uninova.pt/inlife/
} 
diagnostic system scales in highly dynamic environments with a considerable number of nodes.

A common interaction semantic is required to enable consistent diagnosis. In this context, the interactions between the agents have to be characterized. For the problem considered a suitable representation is the following $I=(D, N)$ where $I$ denotes an interaction, $D$ an interaction's direction and $N$ its nature. The system can therefore be envisioned as a directed graph where each agent is a node and each link is a specified interaction. While the nature of the interaction can be any symbol with an associated semantic, the direction is either: inbound, outbound or both $\left(d \in\left\{I_{b}, O_{b}, B\right\}\right)$. Distinct networks are considered for each interaction nature (n).

Internally each agent can be in one of the following states: $\mathrm{OK}$ - the module abstracted by the agent is working normally; NOK - the module has a fault, PFO - the module is propagating a fault, that he has generated, through its outbound connections; PFOther - the module is affected by a propagating fault on its inbound connections and PFOPFOther - the module is being affected by a fault that is propagating through its inbound connections and that it is propagating over it is outbound connections. The last state may raise some confusion of whether the propagated fault is from an inbound neighbour and is transmitting to outbound neighbours or is the summed effect of this interaction with a self-generated fault however, the relevant aspect to consider is the propagation itself. Once the origin of the fault is eliminated the agent will exhibit a state that is either OK or PFO accordingly to the fault's context and disambiguate the previous occurrence as depicted in Fig 1.

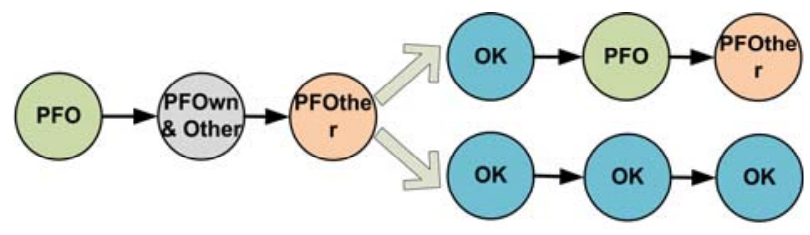

a)

b)

Fig. 1. The agents' states during a fault (a) and after that fault has been resumed in its origin (b) where the system can emerge two distinct configurations according the presence of fault summing effects

Fig. 1 also details a fundamental aspect which is the polarization of the agents' state. The agent's observations enable the emergence of a polarized consensus on the network's state. A note on how the agents perform their observations is worth at this point. Rather than polling information from its direct neighbours they push it asynchronously when their internal state changes.

The quality and type of the observations play a major role on the convergence of the system to a useful diagnosis (the network emerging a consensus for any given fault context). In the next section the present diagnostic method will be formalized using Hidden Markov Models (HMM) and the impact of the observations in ensuring the converge of the network will be assessed in two scenario where the influence of other agents state weighted differently. 


\section{Preliminary Experiments and Results}

The diagnostic model proposed can be formalized as HMM [17] which is the following tuple $\lambda=(A, B, \pi)$. Where $A$ is a $N \times N$ matrix ( $N$ is the number of states in the model) denoting the state transition probabilities $P\left(q_{t+1}=S_{j} \mid q_{t}=S_{i}\right)=a_{i j}, B$ is a $N \times M$ matrix ( $M$ is the number of observation symbols) that encloses the observation probabilities $P\left(O_{t}=k \mid q_{t}=S_{i}\right)=b_{i}(k)$ (i.e. the probability that the observation k happens given the state $S_{i}$ ), $\pi$ represents the starting state probabilities (in the model considered in this paper the initial state of the model is always OK $\mathrm{P}=1$ ). In the present case $N=5$ where each state has the semantic described in the previous section. Concerning $M$, two scenarios are considered $M=8$ (Table 1) and $M=18$ (Table 2). For brevity not all the observation symbols are represented in the second case.

Table 1. Observation alphabet for $M=8$

\begin{tabular}{cccc}
\hline Symbol & Agent Internal State & Inbound Interactions & Outbound Interactions \\
\hline OOO & 0 & 0 & 0 \\
OOF & 0 & 0 & 1 \\
OFO & 0 & 1 & 0 \\
OFF & 0 & 1 & 1 \\
FOO & 1 & 0 & 0 \\
FOF & 1 & 0 & 1 \\
FFO & 1 & 1 & 0 \\
FFF & 1 & 1 & 1 \\
\hline
\end{tabular}

To clarify the reading of the tables in Table 1 the symbol OOF implies that the agent observes that its sensor readings are normal as well as its neighbours with inbound connections. On the contrary, the neighbours with outbound connections are affected with faults. Both models share the A matrix. For brevity both $\mathrm{A}, \mathrm{B}_{1}$ and $\mathrm{B}_{2}$ matrices have been omitted. Table 2 considers more observation symbols that enrich

Table 2. Observation alphabet for $M=18$ note that some of the symbols have been omitted and some combinations denoting simultaneously inbound minority and majority are impossible

\begin{tabular}{cccccc}
\hline Symbol & $\begin{array}{c}\text { Own } \\
\text { Fault }\end{array}$ & $\begin{array}{c}\text { Inbound } \\
\text { Minority }\end{array}$ & $\begin{array}{c}\text { Inbound } \\
\text { Majority }\end{array}$ & $\begin{array}{c}\text { Outbound } \\
\text { Minority }\end{array}$ & $\begin{array}{c}\text { Outbound } \\
\text { Majority }\end{array}$ \\
\hline Ok & 0 & 0 & 0 & 0 & 0 \\
IMaj & 0 & 0 & 1 & 0 & 0 \\
IMin & 0 & 1 & 0 & 0 & 0 \\
OF & 1 & 0 & 0 & 0 & 0 \\
OF_OMin & 1 & 0 & 0 & 1 & 0 \\
OF_IMaj & 1 & 0 & 1 & 0 & 0 \\
OF_IMaj_OMin & 1 & 0 & 1 & 1 & 0 \\
OF_IMin & 1 & 1 & 0 & 0 & 0 \\
OF_IMin_OMaj & 1 & 1 & 0 & 0 & 1 \\
OF_IMin_OMin & 1 & 1 & 0 & 1 & 0 \\
\hline
\end{tabular}


the model and allow agents a finer granularity control over the inbound and outbound observations. Further they allow the proper balance of B matrices supporting changes in opinions as larger number of neighbours are affected by a fault.

To test both models a simple experiment comprising four agents was set up. Two pneumatic valves $\left(\mathrm{V}_{1}\right.$ and $\left.\mathrm{V}_{2}\right)$ controlling the compressed air flow from a robotic manipulator $\left(R_{1}\right)$ which provides air to a gripper $\left(G_{2}\right)$. One of the valves allows air in the gripper and the other expels the air when it is not used as shown in Fig.2. Tables 3 and 4 summarize the sequence of observations each agent performs for the cases where $M=8$ and $M=18$ respectively

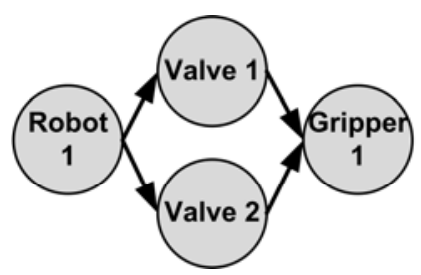

Fig. 2. Network of agents under study

The robot detects a fault using its sensor information. That information is passed on to its direct neighbours $\mathrm{V}_{1}$ and $\mathrm{V}_{2}$. Soon $\mathrm{V}_{1}$ and $\mathrm{V}_{2}$ will also fail causing a change in the agents' observations. In this example the differences in the observations are felt by $\mathrm{G}_{1}$ which in the first model is limited basic inbound fault detection. As shall be seen in Table 5 ignoring minorities and majorities, ultimately the fault context, impacts the final consensus on the network.

Table 3. Sequences of observations per agent with $M=8$ for eight consecutive instants

\begin{tabular}{cccccccccc}
\hline Agent & $\mathrm{t}_{0}$ & $\mathrm{t}_{1}$ & $\mathrm{t}_{2}$ & $\mathrm{t}_{3}$ & $\mathrm{t}_{4}$ & $\mathrm{t}_{5}$ & $\mathrm{t}_{6}$ & $\mathrm{t}_{7}$ & $\mathrm{t}_{8}$ \\
\hline $\mathrm{R}_{1}$ & OOO & FOO & FOO & FOO & FOF & FOF & FOF & FOF & FOF \\
$\mathrm{V}_{1}$ & OOO & OOO & OFO & FFO & FFO & FFO & FFO & FFO & FFO \\
$\mathrm{V}_{2}$ & OOO & OOO & OFO & OFO & FFO & FFO & FFO & FFO & FFO \\
$\mathrm{G}_{1}$ & OOO & OOO & OOO & OFO & OFO & OFO & OFO & OFO & OFO \\
\hline
\end{tabular}

Table 4. Sequences of observations per agent with $M=18$ for eight consecutive instants

\begin{tabular}{cccccccccc}
\hline Agent & $\mathrm{t}_{0}$ & $\mathrm{t}_{1}$ & $\mathrm{t}_{2}$ & $\mathrm{t}_{3}$ & $\mathrm{t}_{4}$ & $\mathrm{t}_{5}$ & $\mathrm{t}_{6}$ & $\cdot$ & $\mathrm{t}_{8}$ \\
\hline $\mathrm{R}_{1}$ & Ok & OF & OF & OF & OF_OMin & OF_OMaj & OF_OMaj &. & OF_OMaj \\
$\mathrm{V}_{1}$ & Ok & Ok & IMin & OF_IMin & OFIMin & OFIMin & OFIMin_OMin &. & OFIMin_OMin \\
$\mathrm{V}_{2}$ & Ok & Ok & IMin & IMin & OFIMin & OFIMin & OFIMin_OMin &. & OFIMin_OMin \\
$\mathrm{G}_{1}$ & Ok & Ok & Ok & Ok & IMin & IMaj & IMaj &.$\cdot$ & IMaj \\
\hline
\end{tabular}

Table 5 clearly illustrates that the $\mathrm{M}=18$ model better captures the fault propagation dynamic in the network. The final result is always biased by the B matrix set up. When learning the model parameters from sample data the second diagnostic model has also the advantage of being able to capture system's details such as the relation 
Table 5. Evolution of agents' internal as new observations are processed

\begin{tabular}{|c|c|c|c|c|c|c|c|c|}
\hline Observations & $\mathrm{t}_{0}$ & $\mathrm{t}_{1}$ & $t_{2}$ & $t_{3}$ & $\mathrm{t}_{4}$ & $t_{5}$ & $\mathrm{t}_{6}$ & $\cdots$ \\
\hline \multicolumn{9}{|l|}{$M=8$} \\
\hline$\overline{\mathrm{R}_{1}}$ & $\overline{\mathrm{Ok}}$ & $\mathrm{NOk}$ & $\mathrm{NOk}$ & $\mathrm{NOk}$ & $\overline{\mathrm{PFO}}$ & $\overline{\mathrm{PFO}}$ & PFO & $\overline{\mathrm{PFO}}$ \\
\hline $\mathrm{V}_{1}$ & Ok & Ok & Ok & PFOther & PFOther & PFOther & PFOther & PFOther \\
\hline $\mathrm{V}_{2}$ & Ok & Ok & Ok & Ok & PFOther & PFOther & PFOther & PFOther \\
\hline $\mathrm{G}_{1}$ & $\mathrm{Ok}$ & $\mathrm{Ok}$ & Ok & $\mathrm{Ok}$ & Ok & Ok & $\mathrm{Ok}$ & Ok \\
\hline \multicolumn{9}{|l|}{$\mathrm{M}=18$} \\
\hline$\overline{\mathrm{R}_{1}}$ & Ok & NOk & $\mathrm{NOk}$ & NOk & PFO & PFO & PFO & PFO \\
\hline $\mathrm{V}_{1}$ & Ok & $\mathrm{Ok}$ & $\mathrm{Ok}$ & PFOther & PFOther & PFOther & PFOPFOther & ... PFOPFOther \\
\hline $\mathrm{V}_{2}$ & Ok & Ok & Ok & Ok & PFOther & PFOther & PFOPFOther & $\cdots$ PFOPFOther \\
\hline $\mathrm{G}_{1}$ & Ok & Ok & Ok & Ok & Ok & PFOther & PFOther & $\cdots \quad$ PFOther \\
\hline
\end{tabular}

between the reliability of the agent's sensor and indirect fault inference (inferring the agent's own fault behaviour considering its neighbours response to faults.). In the present test the first model failed in diagnosing $G_{1}$ as it is highly unlikely that if both valves fail the gripper will remain operational (state $\mathrm{OK}$ ) whether in the second model $\mathrm{G}_{1}$ detected that it was under the effect of fault propagation. If $G_{1}$ had been connected to another device the error would propagate and affect the emerged network consensus.

\section{Conclusions and Future Work}

The presented preliminary experiments testing the proposed diagnostic method suggest that there significant differences in the emergence of coherent diagnostic consensus at network level when ignoring the fault context. Further testing is required to study the behaviour of the proposed method in networks with a high number of interacting agents and distinct levels of connectivity. Also, the fault update dynamics of the agents (propagation of error messages) needs to be addressed to understand of synchronous versus asynchronous update can influence the overall outcome. Although is still early to conclude the results obtained are encouraging and suggest that the proposed method may be suitable to support diagnosis under the framework of the EPS paradigm.

\section{References}

1. Christopher, M.: The Agile Supply Chain: Competing in Volatile Markets. Industrial Marketing Management 29, 37-44 (2000)

2. Camarinha-Matos, L.M., Afsarmanesh, H., Novais, P., Analide, C. (eds.): Establishing the Foundation of Collaborative Networks, vol. 243. Springer, New York (2007)

3. Alsterman, H., Barata, J., Onori, M.: Evolvable Assembly Systems Platforms: Opportunities and Requirements. In: Molfino, R. (ed.) Intelligent Manipulation and Grasping, IMG 2004, Genova, vol. 1, pp. 18-23 (2004)

4. Barata, J., Onori, M., Frei, R., Leitão, P.: Evolvable Production Systems: Enabling Research Domains. In: CARV 2007 - 2nd International Conference on Changeable, Agile, Reconfigurable, and Virtual Production, Toronto - Canada (2007) 
5. Amaral, L.A.N., Ottino, J.M.: Complex networks: Augmenting the framework for the study of complex systems. The European Physical Journal B 38, 147-162 (2004)

6. Newman, M.E.J.: The structure and function of complex networks. SIAM Review 45, 167-256 (2003)

7. Colombo, A.W.: Industrial Agents: Towards Collaborative Production Automation, Management and Organization. IEEE Industrial Electronics Society Newsletter 52, 17-18 (2005)

8. Isermann, R.: Fault Diagnosis Systems: An Introduction from Fault Detection to Fault Tolerance. Springer, Berlin (2006)

9. Venkatasubramanian, V., Rengaswamy, R., Kavuri, S.N.: A review of process fault detection and diagnosis Part III: Process history based methods. Computers and Chemical Engineering 27, 327-346 (2003)

10. Venkatasubramanian, V., Rengaswamy, R., Kavuri, S.N.: A review of process fault detection and diagnosis Part II: Qualitative models and search strategies. Computers and Chemical Engineering 27, 313-326 (2003)

11. Barata, J., Ribeiro, L., Colombo, A.W.: Diagnosis using Service Oriented Architectures (SOA). In: International Conference on Industrial Informatics. IEEE, Vienna (2007)

12. Ribeiro, L.: A Diagnostic Infrastructure for Manufacturing Systems. Electrical and Computer Science Engineering, vol. MSC. New University of Lisbon, Lisbon, 121 (2007)

13. Lopes, G., Pereira, L.M.: Prospective Programming with ACORDA. Empirically Successful Computerized Reasoning, Seattle, USA (2006)

14. Zhou, S., Zhang, J., Wang, S.: Fault diagnosis in industrial processes using principal component analysis and hidden markov models. In: American Control Conference, Boston, USA (2004)

15. Rodrigues, M.A., Liu, Y., Bottaci, L., Rigas, D.I.: Learning and diagnosis in manufacturing processes through an executable Bayesian network. In: 13th international conference on Industrial and engineering applications of artificial intelligence and expert systems: Intelligent problem solving: methodologies and approaches, New Orleans, Louisiana, United States, pp. 390-395 (2000)

16. Son, J.P., Park, J.H., Cho, Y.Z.: An integrated knowledge representation scheme and query processing mechanism for fault diagnosis in heterogeneous manufacturing environments. Robotics and Computer Integrated Manufacturing 16, 133-141 (2000)

17. Lawrence, R.R.: A tutorial on hidden Markov models and selected applications in speech recognition. Readings in speech recognition, pp. 267-296. Morgan Kaufmann Publishers Inc., San Francisco (1990) 